\title{
Taylor Series Based Architecture for Quadruple Precision Floating Point Division
}

\author{
Manish Kumar Jaiswal ${ }^{1}$, and Hayden K.-H So ${ }^{2}$ \\ Dept. of EEE, The University of Hong Kong, Hong Kong \\ Email: ${ }^{1}$ manishkj@eee.hku.hk, ${ }^{2} h s o @ e e e . h k u . h k$
}

\begin{abstract}
This paper presents an area efficient architecture for quadruple precision division arithmetic on the FPGA platform. Many application demands for the higher precision computation (like quadruple precision) than the single and double precision. Division is an important arithmetic, but requires a huge amount of hardware resources with increasing precision, for a complete hardware implementation. So, this paper presents an iterative architecture for quadruple precision division arithmetic with small area requirement and promising speed. The implementation follows the standard processing steps for the floating point division arithmetic, including processing of sub-normal operands and exceptional case handling. The most dominating part of the architecture, the mantissa division, is based on the series expansion methodology of division, and designed in an iterative fashion to minimize the hardware requirement. This unit requires a 114x114 bit integer multiplier, and thus, a FPGA based area-efficient integer multiplier is also proposed with better design metrics than prior art on it. These proposed architectures are implemented on the Xilinx FPGA platform. The proposed quadruple precision division architecture shows a small hardware usage with promising speed.
\end{abstract}

Keywords-Floating Point Arithmetic, Division, FPGA, Iterative Architecture, FSM, Taylor Series Expansion Division, Digital Arithmetic.

\section{INTRODUCTION}

Floating point arithmetic is an integral part of scientific and engineering applications. The single precision (SP) floating point arithmetic supports roughly 7 significant decimal digits, whereas, the double precision (DP) supports roughly for 15 decimal digits. The precision requirements for a large set of applications are well with the reach of single precision and double precision computation. However, a significant number of important applications need a higher precision computation [1], [2], that can be suffice by the quadruple precision $(\mathrm{QP})$ arithmetic which provides roughly 30 decimal digits precision.

The software solution for the quadruple precision are much slower [2]. So, in the absence of direct hardware support for quadruple precision arithmetic on contemporary processing systems, another better alternative would be the use of reconfigurable platform. FPGA (Field Programmable Gate Arrays) based reconfigurable platforms are well explored during last couple of decades. A large set of applications from various domains, ranging from scientific and numerical processing, image and signal processing, cryptography, communications, bio-medical application etc are well experimented on the FPGA devices.

Literature contains a limited work on the FPGA based quadruple precision (high precision) arithmetic architectures [3], [4], [5], which aimed for floating point multiplication arithmetic. The work by Diniz et al. [6] has presented the design metrics for the quadruple precision division, but without any architectural and implementation details. Literature lacks for the efficient architecture for the FPGA based quadruple precision division arithmetic, which is a complex arithmetic than adder and multiplier. Although, many researchers have demonstrated the architectures for (up to) double precision division[7], [8], [9], [10] but their direct extension for higher precision (beyond double precision) are often impractical due to large area/look-up-table requirements.

In this view, this work is aimed towards an architecture for quadruple precision division arithmetic on FPGA platform, moreover, similar strategy can be easily built for any higher precision division implementation. This paper used a multiplicative based division methodology, the series expansion method [9], for the underlying mantissa division. The multiplicative based methods provide higher performance than conventional digit-recurrence method (eg. SRT method). Other multiplicative based methods (Newton Raphson, Goldschmidt [11]) can also be built using similar strategy. Moreover, since the complete hardware requirement for the quadruple precision arithmetic is large, and the division is not so frequent than addition/multiplication arithmetic, an iterative architecture is proposed for the quadruple precision floating division arithmetic on FPGA device.

The main contributions of present work can be summarized as follows:

- Proposed a quadruple precision floating point division architecture on a reconfigurable FPGA platform. It includes the computational support for normal as well as sub-normal operands, along with all the exceptional case handling. Architecture is fully pipelined, and designed in iterative fashion for area-efficiency.

- An efficient mantissa division architecture is designed using series expansion methodology of division. It comprised of a finite state machine (FSM) which schedules the data over a large integer multiplier iteratively to compute the mantissa division.

- For mantissa division architecture, an area efficient $114 \times 114$ bit integer multiplier is designed which 
is specifically aimed for FPGA platform, and outperforms prior literature work on it.

\section{BACKGROUND}

The quadruple precision is a 128-bit format of IEEE floating point standard [12]. The standard format for the quadruple precision floating point number is as below.

$$
\overbrace{\text { Sign-bit }}^{1-\text { bit }} \overbrace{\text { exponent }}^{15-\text { bit }} \overbrace{\text { mantissa }}^{112-\text { bit }}
$$

The computational flow for floating point division arithmetic is shown in Algo 1. This algorithm provides computational support for both, the normal and sub-normal processing. It also comprised of exceptional case handling (infinity, $\mathrm{NaN}$ ) and their processing.

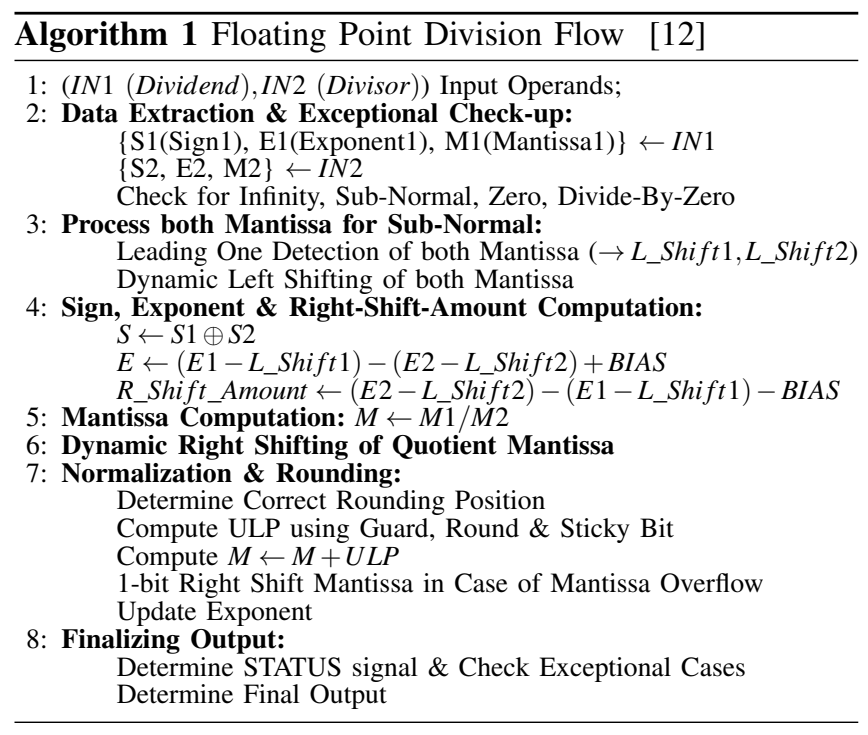

\section{A. Mantissa Division Methodology}

The most critical part of the above algorithm is the mantissa division implementation. Here, the underlying methodology for this computation is discussed. It is based on the series expansion method of division, as follows.

Let $m_{1}$ be the normalized dividend mantissa, $m_{2}$ be the normalized divisor mantissa and $q$ will be the mantissa quotient, which can be computed as follows,

$$
q=\frac{m_{1}}{m_{2}}=\frac{m_{1}}{a_{1}+a_{2}}=m_{1} \times\left(a_{1}+a_{2}\right)^{-1}
$$

Here, the divisor mantissa $m_{2}$ is partitioned in to two part as $a_{1}$ (W-bit) and $a_{2}$ (all remaining bits) as below.

$$
m_{2} \rightarrow \overbrace{1 . \underbrace{x x x x x x x x x}_{W}}^{a_{1}} \overbrace{x x \ldots \ldots \ldots . . \ldots x x x x x x}^{a_{2}}
$$

By using Taylor Series expansion,

$$
\left(a_{1}+a_{2}\right)^{-1}=a_{1}^{-1}-a_{1}^{-2} \cdot a_{2}+a_{1}^{-3} \cdot a_{2}^{2}-a_{1}^{-4} \cdot a_{2}^{3}+\cdots
$$

In above equation, the pre-computed value of $a_{1}^{-1}$ can be used (from pre-stored look-up table) to perform the entire computation, by using integer multipliers, adders and subtractor; which are easily realizable in hardware implementation. The pre-computed value of $a_{1}^{-1}$ acts as an initial approximation for $m_{2}^{-1}$, which further improved with remaining computation. Here, the size $W$ (bit width) of $a_{1}$ (here, the hidden ' 1 ' bit place in $a_{1}$ is not counted, as it remains as constant value in the normalized format) determines the size of memory (for look-up table to store $a_{1}^{-1}$ ) and the number of terms from the series expansion, to perform the computation for a given precision. The number of terms $(N)$ (with a given $W$ ) for the quadruple precision (precision requirement $2^{-113}$ ) can be determined by following inequality:

$$
\begin{aligned}
\left|E_{N}\right| & =\left|a_{1}^{(N+1)} \cdot a_{2}^{N}\left(1-a_{1}^{-1} \cdot a_{2}+a_{1}^{-2} \cdot a_{2}^{2}-a_{1}^{-3} \cdot a_{2}^{3}-\ldots\right)\right| \\
& =\left|\frac{a_{1}^{(N+1)} \cdot a_{2}^{N}}{1+a_{1}^{-1} \cdot a_{2}}\right| \leq 2^{-113}
\end{aligned}
$$

where, $E_{N}$ is error caused by all the ignored terms in eq.2. For maximum error, numerator of eq. 3 should be maximum with the minimum value for numerator. So, for most pessimistic estimation (for minimum denominator, let $\left(1+a_{1}^{-1} \cdot a_{2}\right) \approx 1$, and for maximum numerator let $\left.a_{1}^{-1}=1\right)$,

$$
\left|E_{N}\right|=\left|a_{2}^{N}\right| \leq 2^{-113}
$$

Thus, it can seen that for a given precision requirement, increase in $W$ would reduces the required terms $N$ and vice-versa. Here, the value of $W$ determines the size of memory (to store the pre-computed $a_{1}^{-1}$ ), and $N$ determines the amount of other hardwares (multipliers, adders, subtractors). For, a good balance, bit width of $W=8$ for $a_{1}$ is selected, which requires seventeen terms (up to $a_{1}^{-17} \cdot a_{2}^{16}$ ) to meet the requirement of quadruple precision computation. The final quotient is simplified and expressed as below, in order to optimize the hardware resource and latency of the computation.

$$
\begin{aligned}
q=m_{1} & \cdot a_{1}^{-1}-m_{1} \cdot a_{1}^{-1}\left\{\left(a_{1}^{-1} \cdot a_{2}-a_{1}^{-2} \cdot a_{2}^{2}\right)\right. \\
& \times\left(1+a_{1}^{-2} \cdot a_{2}^{2}+a_{1}^{-4} \cdot a_{2}^{4}+a_{1}^{-6} \cdot a_{2}^{6}\right) \times\left(1+a_{1}^{-8} \cdot a_{2}^{8}\right\}
\end{aligned}
$$

The size of look-up table to store $a_{1}^{-1}$ is taken as $2^{8} \times 113$, which provides sufficient precision for remaining computations. Also, a full multiplier of size $114 \times 114$-bit is used iteratively for computing all the terms, which helps in preserving the accuracy of all the terms[7], [13].

\section{Proposed QuAdruple PRECISION DIVISION ARCHITECTURE}

The proposed division architecture is shown in Fig. 1. Here, it composed of three stages. The details of each stage architecture is discussed below in following subsections oneby-one. 


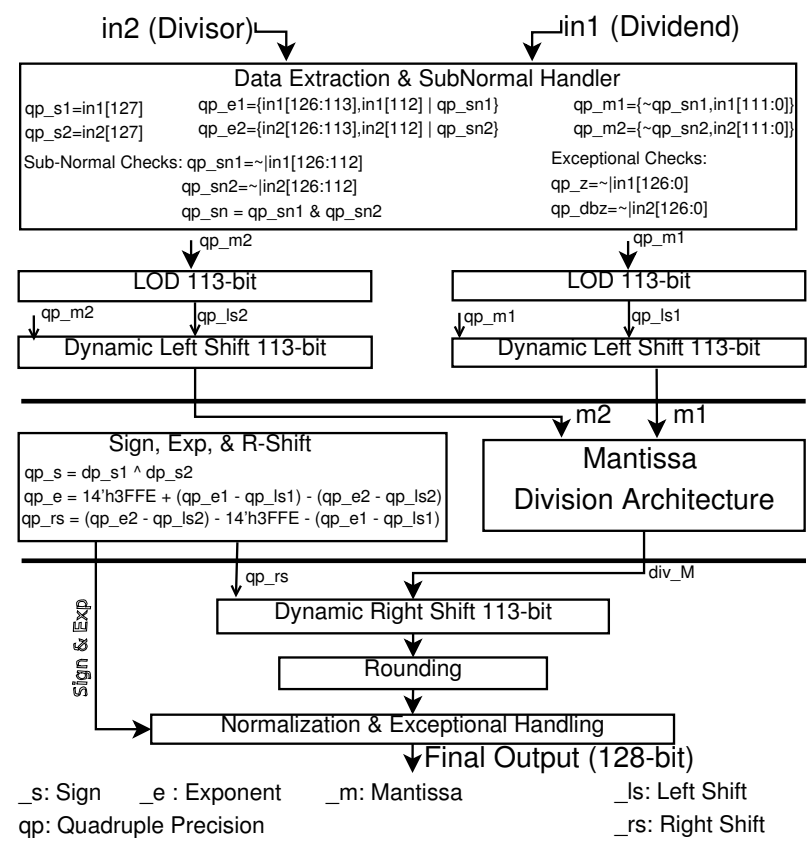

Figure 1: Division Architecture

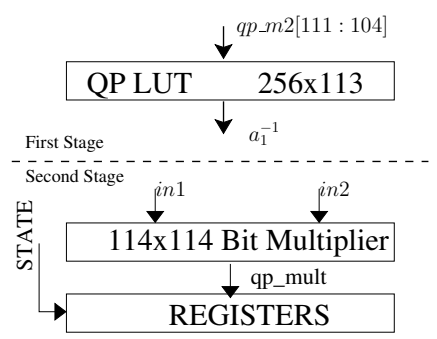

Figure 2: QP Mantissa Division Architecture

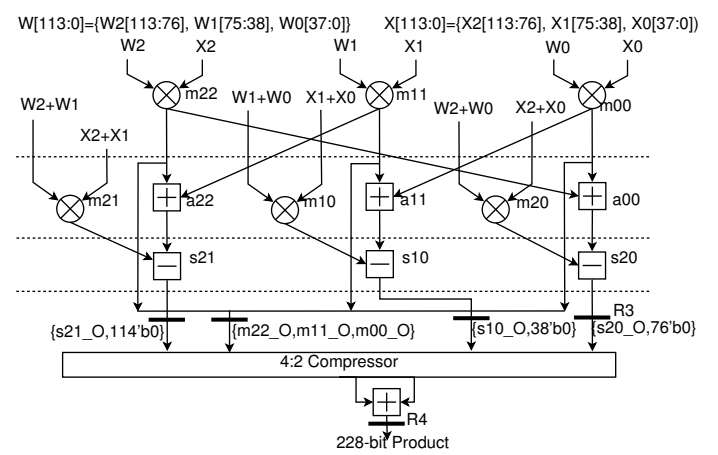

Figure 3: 114x114 Integer Multiplier Architecture

1) Mantissa Division Unit: The mantissa computation is the most complex part of the floating point division arithmetic. This architecture includes the implementation of eq.(5), and is shown in Fig. 2. It includes a look-up table to pre-store the initial inverse approximation of divisor mantissa (in first-stage), and the remaining computation is built around a $114 \times 114$ bit integer multiplier, in an iterative fashion. A finite state machine (FSM) is designed which decides the effective inputs for the multiplier in each state, the details for which are discussed after the description for the architecture of 114x114 bit multiplier.

2) Multiplier Architecture: A 114x114 bit integer multiplier is designed using combination of 3-partition and 2partition Karatsuba method [14].First, it is partitioned in to three sets of 38-bit, which requires three multiplier blocks of $38 \times 38$ and three of size $39 \times 39$. For this, 39x39 multiplier is designed, and also used as $38 \times 38$ multiplier. The $39 \times 39$ multiplier is designed using two partition method, which need three blocks of multipliers (one 19x19, one 20x20 and one $21 \times 21$ ). A $21 \times 21$ multiplier is designed using a DSP48E block on Xilinx FPGA (available on Virtex-5 onward series) and some logics. These architectures are shown in Fig. 3 and Fig. 4 for $114 \times 114$ and 39x39 multiplier, respectively.

Three pipelined version of the $114 \times 114$ multiplier is used for the purpose of mantissa division; 1-stage, 3-stage and 6-stage multiplier. This is to discuss the trade-off among latency-throughput-performance-area. The multiplier architectures are presented with 5 level pipeline registers (R0-R5), which forms a 6-stage multiplier (with registered inputs). For 


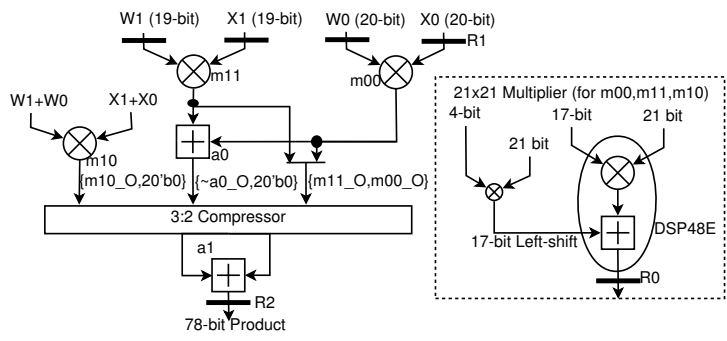

Figure 4: 39x39 Integer Multiplier Architecture

3-stage multiplier, only R0 and R3 are present, and R1, R2, and $\mathrm{R} 4$ are absent, whereas for 1-stage, none of pipeline registers are present.

The interesting point of the presented $114 \times 114$ multiplier is that, it requires only 18 DSP48E blocks (3 for each 39x39), whereas, using traditional method it need 49 DSP48E blocks.

3) Mantissa Division Finite State Machine: An iterative mantissa division is designed to have an area efficient architecture. The architecture is based on the hardware realization of eq.(5), which is listed below for an easy reference.

$$
\begin{aligned}
& m_{1} \cdot a_{1}^{-1}-m_{1} \cdot a_{1}^{-1}\left\{\left(a_{1}^{-1} \cdot a_{2}-a_{1}^{-2} \cdot a_{2}^{2}\right)\right. \\
& \quad \times\left(1+a_{1}^{-2} \cdot a_{2}^{2}+a_{1}^{-4} \cdot a_{2}^{4}+a_{1}^{-6} \cdot a_{2}^{6}\right) \times\left(1+a_{1}^{-8} \cdot a_{2}^{8}\right\}
\end{aligned}
$$

Here, $m_{1}$ is the normalized dividend mantissa; and $m_{2}$ is the normalized divisor mantissa. Here, $m_{2}$ is further partitioned into $a_{1}$ (first 8-bit right to the decimal point) and $a_{2}$ (all remaining bits right to the $a_{1}$ ).

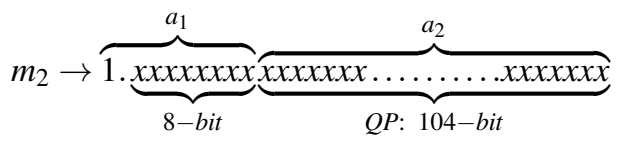

Here, for the ease of understanding and better readability of the later description, various combinations of terms in above equation are listed as follows:

$$
\begin{array}{rlrl}
A & =m_{1} \cdot a_{1}^{-1} & B & =a_{1}^{-1} \cdot a_{2} \\
C & =B^{2}=a_{1}^{-2} \cdot a_{2}^{2} & D & =B^{4}=C^{2}=a_{1}^{-4} \cdot a_{2}^{4} \\
E & =B^{6}=C^{3}=a_{1}^{-6} \cdot a_{2}^{6} & F & =B^{8}=C^{4}=a_{1}^{-8} \cdot a_{2}^{8} \\
G & =B-C=a_{1}^{-1} \cdot a_{2}-a_{1}^{-2} \cdot a_{2}^{2} & H_{T} & =1+C+D \\
H & =H_{T}+F & & =1+a_{1}^{-2} \cdot a_{2}^{2}+a_{1}^{-4} \cdot a_{2}^{4} \\
& =1+a_{1}^{-2} \cdot a_{2}^{2}+a_{1}^{-4} \cdot a_{2}^{4}+a_{1}^{-6} \cdot a_{2}^{6} & I & =1+F=1+a_{1}^{-8} \cdot a_{2}^{8} \\
J & =G \cdot H & K & =J \cdot I \\
L & =A . K & M & =A-L
\end{array}
$$

The implementation is achieved by designing a FSM. First, we will discuss the FSM with 1-stage multiplier, which consists of 11 states(S0 to S10), and later explore with multistage multiplier. In each state of FSM, inputs (in 1 and in 2 ) for the $114 \times 114$ multiplier is determined, and its output is assigned to the designated terms, which proceeds as follows:

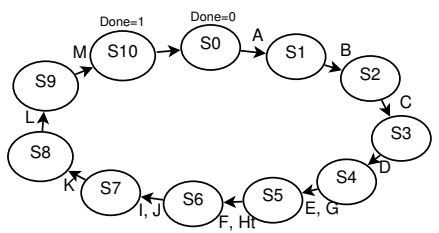

Figure 5: Mantissa Division FSM (With 1-Stage Multiplier)

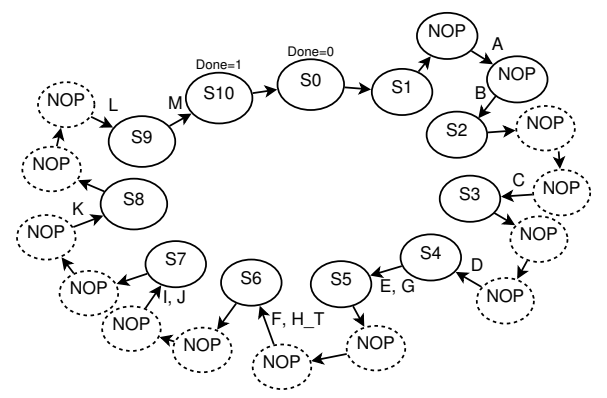

Figure 6: Mantissa Division FSM (With 3-Stage Multiplier)

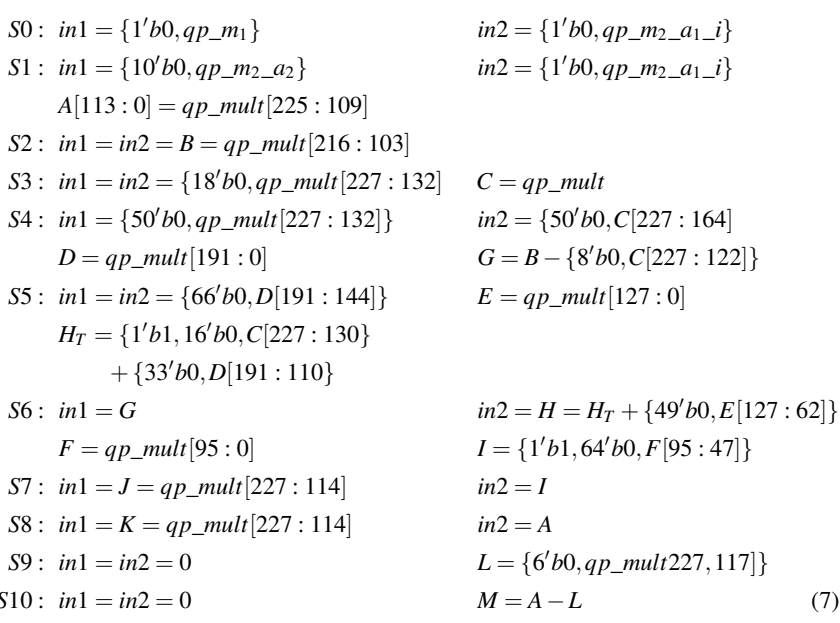

The finite state machine (FSM) (with 1-stage multiplier) is shown in Fig. 5. The selection of bits for a term is based on the position of the decimal point. Generally, the multiplications are done in 114-bit (sufficient for it's precision requirement) and add/sub are performed in 128-bit (to preserve precision). The mantissa division FSM requires 11 cycles for processing, with 1-stage multiplier.

Performance can improve by pipelining the multiplier, which asks for suitable modification in the mantissa division FSM. It can be easily achieved by inserting the NOP (NoOperation state) between two states, where the output of multiplier from previous state acts as the input of multiplier in next state. For the case of FSM with 3-stage multiplier, it is shown in Fig. 6, where it requires to insert two NOP states for each of the above discussed case. It is presented over the '1-stage multiplier FSM' for better understanding 
of where to perform the insertion of the NOP states. Thus, it requires 25 states with three stage multiplier. Similarly, it is formed with a 6-stage multiplier by inserting 5-NOP states at the similar instances, and it requires a total of 47 states, (similarly, it can be designed with any multiple stage multiplier).

\section{Stage-3:Normalization, Rounding and Post-processing}

The third stage of the FP division architecture corresponds to the computations of steps 6,7 and 8 of the Algo 1. In this stage, for the case of exponent underflow (if dividend exponent is smaller than the divisor exponent), mantissa division quotient is first process for the dynamic right shifting (needs 7, 113-bit, 2:1 MUXs). Which is followed by the rounding round-to-nearest of the quotient mantissa, and then it undergoes normalization and exceptional case processing, and final updates of exponent and mantissa.

\section{IMPLEMENTATION RESULTS \& COMPARISONS}

The proposed quadruple precision division architecture is implemented and placed \& routed on various Xilinx FPGA Devices (Virtex-5, Virtex-6 and Virtex-7) are used for the implementation. The implementation details are shown in Table-I. In the case of division architecture with 6-stage multiplier, the first-stage and third-stage of division architecture (in Fig. 1) are also pipelined by one extra level to meet the critical path of the multiplier. However, with the 3-stage multiplier, they are in single stage. Thus, the latency of the QP division architecture with 1-stage multiplier becomes 13 cycles (1 cycle first-stage, 11-cycles FSM, and 1 cycle thirdstage) with throughput of 12 cycles (after every first-stage and FSM processing). Similarly, with 3-stage multiplier, the latency become 27 cycles and throughput becomes 26 cycles. Likewise, with 6-stage multiplier division architecture, the latency is 50 cycles ( 2 cycle first-stage, 46 cycles FSM and 2 cycles third-stage) and throughput is 48 cycles. The inclusion of multi-stage multiplier in the mantissa division clearly improves the speed of the architecture, however, it decreases the throughput, with minor changes in LUTs counts, and additional FFs for pipeline registers. It is also obvious to see the improvements in speed with the use of higher grade FPGA devices, from Virtex-5 to Virtex-7.

The proposed method is able to produce faithful rounded result. Faithful rounding result is suitable for a large set of applications. However, correctly rounded result can be obtained by processing one more full multiplication [7], [13], which adds few more cycles count in FSM. The functional verification of the proposed architecture is also carried out using 5-millions random test cases for each of the normal-normal, normal-subnormal, subnormal-normal and subnormal-subnormal operands combination, along with the other exceptional case verification, which produces a maximum of 1-ulp (unit at last place) precision loss.
Table I: Implementation Details

\begin{tabular}{|l|c|c|c|c|}
\hline \multicolumn{2}{|l|}{ Multiplier-Pipeline (MP) } & MP-1 & MP-3 & MP-6 \\
\hline \multicolumn{2}{|l|}{ Latency (cycle) } & 13 & 27 & 50 \\
\hline \multicolumn{2}{|l|}{ Throughput (cycle) } & 12 & 26 & 48 \\
\hline \multirow{3}{*}{ LUTs } & Virtex-5 & 7198 & 7168 & 7333 \\
& Virtex-6 & 7232 & 7370 & 7228 \\
& Virtex-7 & 7220 & 7437 & 7229 \\
\hline \multirow{3}{*}{ FFs } & Virtex-5 & 1734 & 2287 & 4355 \\
& Virtex-6 & 2298 & 2677 & 4405 \\
\hline DSP48E & Virtex-7 & 2002 & 2683 & 4397 \\
\hline \multirow{3}{*}{ Freq (MHz) } & Virtex-5 & 64.2 & 128.9 & 172.4 \\
& Virtex-6 & 86.2 & 169.3 & 224.7 \\
& Virtex-7 & 92.7 & 183 & 236 \\
\hline
\end{tabular}

\section{A. Comparisons and Related Discussion}

Diniz et al. [6] has shown the results for a quadruple precision division implementation, but, without any architectural and mantissa division methodology details. Their design usages a large amount of hardware resources (26811 LUTs, 13809 FFs), with latency of 118 cycles at $50 \mathrm{MHz}$ speed.

To the best of author's knowledge, there is no other quadruple precision division architecture on FPGA is available in the literature. So, a discussion based on the methods used in some double precision (DP) implementation is provided here. A SRT-based digit-recurrence double precision division architecture is proposed by Hemmert et al. [15] with 62 cycles latency and 4100 slices, and thus, for quadruple precision, this method would need a large latency and area. However, performance of digit-recurrence method lacks behind multiplicative methods.

Antelo et al. [16] has proposed a combination digitrecurrence approximation and Newton-Raphson (NR) iteration, and its implementation for double precision requires an address space of 15-bit (approx 28 18k BRAMs), and an equivalent of 29 MULT18x18 IPs. Pasca et al. [7] has proposed a combination of polynomial approximation and NR method on an Altera Stratix V device, which usages roughly 1000 ALUTs (ALUTs on Stratix is computationally richer that Xilinx LUTs) and an Xilinx equivalent of 4 BRAMs and 35 multiplier IPs. Wang et al. [17] has reported a 41-bit (10-bit exp and 29-bit mantissa) floating point format division architecture. It requires a large area with 62 BRAMs, and reported to have precision loss. This method requires a huge look-up table with address space of half size of operands, ie $2^{27}$ for DP and $2^{57}$ for QP. Another DP division implementation presented by Fang et al. [10] is based on an initial approximation with Goldschmidt method. An extension of this method for QP division needs a look-up table with address space of $2^{29}$, and some multipliers. Thus, in view of huge look-up table requirements, above methods appears impractical for quadruple precision implementation.

The proposed architecture of $114 \times 114$ bit integer multiplier is also compared here with some recent proposals. 
Banescu et al. [3] has designed a 113x113 multiplier using tiling method which requires 2070 LUTs, 2062 FFs, and 34 DSP48E with a latency of 13 cycles and $407 \mathrm{MHz}$ speed, on Virtex-5 FPGA. Similarly, in [4] a 113x113 bit multiplier architecture requires 2373 slices and 24 DSP48E with a latency of 14 cycles and $310 \mathrm{MHz}$ speed. However, the proposed 6-stage 114x114 integer multiplier architecture requires 3319 LUTs, $2185 \mathrm{FFs}$, and 18 DSP48E, with a latency of 6 cycles and $172 \mathrm{MHz}$ speed, on a Virtex-5 device. On taking account of LUTs equivalent of a DSP48E (for " $(a[23: 0] * b[16: 0])+c[47: 0]$ " operation, in a scope of current usage), a simple synthesis results into 644 LUTs. Thus, the proposed $114 \times 114$ bit multiplier improves on overall equivalent area requirements, provides better DSP48E utilization, while speed can be improved by more pipelines.

\section{CONCLUSions}

This paper presented an iterative architecture for the quadruple precision floating point division arithmetic on reconfigurable FPGA platform. This architecture provides computational support for the normal \& subnormal operands, with processing of various exceptional cases, and produces faithful rounded results. The mantissa division architecture is designed using the series expansion methodology of division operation, which is a multiplicative based division method. For this, an area efficient architecture for $114 \times 114$ integer multiplier is also proposed, which requires small number of DSP48E blocks compared to the previous literature works. The division architecture is explored with various stage multiplier unit, to see the trade-off among area, speed, latency and throughput. The proposed division architecture will leads to the exploration of applications with high precision requirement, on the reconfigurable FPGA platforms.

\section{ACKNOWLEDGMENTS}

This work is party supported by the "The University of Hong Kong" grant (Project Code. 201409176200), the "Research Grants Council" of Hong Kong (Project ECS 720012E), and the "Croucher Innovation Award" 2013.

\section{REFERENCES}

[1] F. de Dinechin and G. Villard, "High precision numerical accuracy in physics research," Nuclear Instruments and Methods in Physics Research Section A: Accelerators, Spectrometers, Detectors and Associated Equipment, vol. 559, no. 1, pp. 207210, 2006.

[2] D. H. Bailey, R. Barrio, and J. M. Borwein, "High-precision computation: Mathematical physics and dynamics," Applied Mathematics and Computation, vol. 218, no. 20, pp. $10106-$ $10121,2012$.

[3] S. Banescu, F. de Dinechin, B. Pasca, and R. Tudoran, "Multipliers for floating-point double precision and beyond on FPGAs," SIGARCH Comput. Archit. News, vol. 38, pp. 73-79, Jan 2011.
[4] M. K. Jaiswal and R. C. C. Cheung, "Area-Efficient Architectures for Large Integer and Quadruple Precision Floating Point Multipliers," in The 20th Annual IEEE Symposium on Field-Programmable Custom Computing Machines. Los Alamitos, CA, USA: IEEE Computer Society, 2012, pp. 2528 .

[5] Y. Dou, Y. Lei, G. Wu, S. Guo, J. Zhou, and L. Shen, "FPGA accelerating double/quad-double high precision floating-point applications for ExaScale computing," in ICS '10: Proceedings of the 24th ACM International Conference on Supercomputing. New York, NY, USA: ACM, 2010, pp. 325-336.

[6] P. Diniz and G. Govindu, "Design of a field-programmable dual-precision floating-point arithmetic unit," in Field Programmable Logic and Applications, 2006. FPL '06. International Conference on, Aug 2006, pp. 1-4.

[7] B. Pasca, "Correctly rounded floating-point division for dspenabled fpgas," in Field Programmable Logic and Applications (FPL), 2012 22nd International Conference on, aug. 2012, pp. $249-254$.

[8] X. Wang and M. Leeser, "Vfloat: A variable precision fixedand floating-point library for reconfigurable hardware," $A C M$ Trans. Reconfigurable Technol. Syst., vol. 3, no. 3, pp. 16:116:34, Sep. 2010.

[9] M. Jaiswal, R. Cheung, M. Balakrishnan, and K. Paul, "Series expansion based efficient architectures for double precision floating point division," Circuits, Systems, and Signal Processing, vol. 33, no. 11, pp. 3499-3526, 2014.

[10] X. Fang and M. Leeser, "Vendor Agnostic, High Performance, Double Precision Floating Point Division for FPGAs," in The 17th IEEE High Performance Extreme Computing (HPEC), Waltham, MA, 2013.

[11] R. E. Goldschmidt, "Application of division by convergence," Master's thesis, Massachusetts Institute of Technology, June 1964.

[12] "IEEE standard for floating-point arithmetic," IEEE Std 7542008, pp. 1-70, Aug 2008.

[13] S. F. Obermann and M. J. Flynn, "Division algorithms and implementations," Computers, IEEE Transactions on, vol. 46 , no. 8, pp. 833-854, Aug. 1997.

[14] A. Karatsuba and Y. Ofman, "Multiplication of Many-Digital Numbers by Automatic Computers," in Proceedings of the USSR Academy of Sciences, vol. 145, 1962, pp. 293-294.

[15] K. S. Hemmert and K. D. Underwood, "Floating-point divider design for FPGAs," IEEE Trans. Very Large Scale Integr. Syst., vol. 15, no. 1, pp. 115-118, 2007.

[16] E. Antelo, T. Lang, P. Montuschi, and A. Nannarelli, "Low latency digit-recurrence reciprocal and square-root reciprocal algorithm and architecture," in 17th IEEE Symposium on Computer Arithmetic, Jun. 2005, pp. 147-154.

[17] X. Wang and M. Leeser, "Vfloat: A variable precision fixedand floating-point library for reconfigurable hardware," $A C M$ Trans. Reconfigurable Technol. Syst., vol. 3, no. 3, pp. 16:1-16:34, Sep. 2010. 